\title{
The Effect of Moderate Weight Loss on a Non-Invasive Biomarker of Liver Fibrosis: A Randomised Controlled Trial
}

\author{
Dimitrios A. Koutoukidis ${ }^{a, b}$ Susan A. Jebb ${ }^{a, b} \quad$ Paul Aveyard ${ }^{a, b}$ \\ Nerys M. Astbury ${ }^{a, b}$ \\ a Nuffield Department of Primary Care Health Sciences, University of Oxford, Oxford, UK;

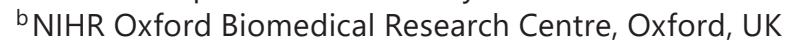

\section{Keywords}

Weight loss $\cdot$ Liver fibrosis · Biomarkers

\begin{abstract}
Background: Referral to weight loss programmes is the only effective treatment for nonalcoholic fatty liver disease (NAFLD). Clinicians should advise weight loss and screen for liver fibrosis using the Enhanced Liver Fibrosis (ELF) score. Aim: To examine if the ELF score changes with weight loss. Design and Setting: Randomised controlled trial (ISRCTN85485463) in UK primary care during 2007-2008. Method: Adults with a BMI of $27-35 \mathrm{~kg} / \mathrm{m}^{2}$ and $\geq 1$ risk factor for obesity-related disease were randomised to attend a community weight loss programme $(n=45)$ or receive usual weight loss advice from a practice nurse $(n=28)$. Weight and the ELF score were measured at baseline and 1 year. Analysis of covariance examined mean changes in the ELF score between groups and its relationship with weight loss. Results: Mean (SD) BMI was $31.10 \mathrm{~kg} / \mathrm{m}^{2}$ (2.55) with evidence of moderate levels of liver fibrosis at baseline (mean ELF score: 8.93 [0.99]). There was no evidence that the community weight loss programme reduced the ELF score compared with usual care (difference +0.13 points, $95 \%$ $\mathrm{Cl}:-0.25$ to 0.52 ) despite greater weight loss (difference: $-2.66 \mathrm{~kg}, 95 \% \mathrm{Cl}:-5.02$ to -0.30 ). Mean weight loss in the whole cohort was 7.8\% (5.9). There was no evidence of an association between weight change and change in ELF; the coefficient for a $5 \%$ weight loss was -0.15 (95\% Cl: -0.30 to 0.0002). Conclusion: We found no evidence that the ELF score changed meaningfully following moderate weight loss. Clinicians should not use the ELF score to measure improvements in NAFLD fibrosis following weight loss programmes.
\end{abstract}


Koutoukidis et al.: Weight Loss on Enhanced Liver Fibrosis Score

\section{Introduction}

Non-alcoholic fatty liver disease (NAFLD) covers a range of conditions from excess fat in theliver through inflammation and fibrosis (non-alcoholic steatohepatitis, NASH), to advanced fibrosis, and cirrhosis. It is estimated that about $25 \%$ of adults worldwide have NAFLD [1]. Among people with obesity, about $50-75 \%$ are also affected by NAFLD [2]. Obesity is associated with more severe forms of the disease and with a worse prognosis for people with NAFLD [3]. Obesity and insulin resistance are associated with the development and progression of NAFLD with NAFLD being widely regarded as the hepatic manifestation of the metabolic syndrome [4]. The incidence of cardiovascular disease in people with severe NAFLD is 2.5-fold higher compared with matched controls [5]. Hepatocellular carcinoma associated with NAFLD has increased 10-fold, and liver transplantation due to NASH cirrhosis has increased 2-fold in the last decades [6, 7].

The high prevalence of NAFLD and its association with an increased risk of morbidity and mortality render it a serious and costly condition. For people with NAFLD, the presence of fibrosis is the strongest predictor of long-term outcomes. Advanced fibrosis is associated with a 14 times higher risk of liver events and three times higher risk of premature mortality $[8,9]$. There is a need to identify methods to measure fibrosis to inform the prognosis and aid clinical decision making about the choice of emerging therapies.

Currently, fibrosis can only be reliably assessed with a liver biopsy. However, even with biopsies there are methodological limitations including sampling error and inter- and intraobserver variability. In addition, the cost, risk of complications, and low patient acceptability means that biopsies cannot be repeated often or in large samples [10]. The Enhanced Liver Fibrosis (ELF) score is emerging as a promising blood biomarker for fibrosis, as it has excellent accuracy for diagnosing histologically confirmed advanced fibrosis with an area under the curve of 0.90 in people with NAFLD and its specificity and sensitivity increasing with increases in the cut-off value $[11,12]$. It has been found to have the highest diagnostic accuracy for advanced fibrosis compared with other non-invasive biomarkers, and, therefore, it is recommended for diagnosing advanced fibrosis in people with NAFLD by the National Institute for Health and Care Excellence (NICE) [13]. Furthermore, its use as a NAFLD screening tool by clinicians has led to appropriate stratification of NAFLD patients and reduced unnecessary referrals to secondary care [14].

There is, however, less certainty that ELF can be used to monitor the progress or improvement in NAFLD. An observational study of a weight loss programme in children with obesity was associated with reductions in ELF at the end of the programme, but $23 \%$ of children with reductions in their BMI-SDS score showed increases in ELF [15]. In a randomised controlled trial of liraglutide, a GLP-1 agonist, among people with NASH, there was a weight loss of $4.7 \mathrm{~kg}$ at 1 year compared with placebo, which was associated with a -0.4-point decrease in the ELF score [16]. However, it is unclear whether this is attributable to the weight loss or a specific effect of the enhanced insulin secretion. Accordingly, there is no evidence that ELF is responsive to change following weight loss through lifestyle modification, the recommended and most commonly advised treatment for NAFLD [13, 17], and it is unclear whether this would be a suitable outcome measure in trials of lifestyle interventions.

Community weight loss groups lead to similar weight loss to liraglutide at a fraction of the cost and are among the most commonly used weight loss programmes. The aim of the current study was to examine whether a community weight loss programme reduces ELF score over 12 months compared with a weight-loss intervention which is less effective. In observational analyses, we examined whether greater weight loss was associated with greater change in ELF score and whether the association was stronger for those with higher ELF scores and therefore more fibrosis at baseline. 
Koutoukidis et al.: Weight Loss on Enhanced Liver Fibrosis Score

\section{Methods}

Participants and Setting

Thiswasasecondaryanalysisofapublishedrandomised controlledtrial(ISRCTN85485463) [18]. Adults with a BMI of $27-35 \mathrm{~kg} / \mathrm{m}^{2}$ and at least one other risk factor for metabolic syndrome or obesity-related disease were recruited from Germany, Australia, and the UK. This analysis included only the UK participants with blood samples at baseline and 1 year $(n=73)$.

\section{Interventions}

Participants were equally randomised to a community weight loss programme (WeightWatchers) or usual care. The WeightWatchers weight loss programme comprised of weekly group meetings over a 12-month period during which participants were weighted and received support and motivation. Participants were advised to follow a hypo-energetic diet based on healthy eating principles using a "points" system equating to about 1,100-1,500 kcal/day. Participants were encouraged to aim for at least 150 min of moderate intensity physical activity weekly. Participants in the usual care group received regular weight loss advice and support from a primary care practitioner.

\section{Assessments}

Weight was measured with calibrated scales, and glucose and insulin were assessed from fasted blood samples. The ELF score was measured in serum and automatically computed by the analyser (ADVIA Centaur XP, Siemens Healthcare Diagnostics) based on the following algorithm combining hyaluronic acid, propeptide of type III procollagen, and tissue inhibitor of metallo-proteinases- $1:$ ELF $=2.278+0.851 \ln (\mathrm{HA})+0.751 \ln ($ PIIINP) $+0.394 \ln$ (TIMP1) . The ELF score was interpreted as none/mild fibrosis for values below 7.7, moderate fibrosis for values between 7.7 and 9.7, and severe fibrosis for values of at least 9.8 [19].

\section{Analysis}

To analyse the difference in ELF between trial arms, we used analysis of covariance with a term for trial arm and baseline ELF score. We examined whether the effect of treatment on ELF score depended upon baseline ELF by adding a multiplicative interaction term between baseline ELF and trial arm.

We also conducted an observational analysis of the relationship between changes in weight and the ELF score at 1 year using general linear regression adjusting for baseline values. We examined whether the association between weight loss and change in ELF was larger for those with higher baseline ELF scores by adding a multiplicative interaction term between baseline ELF and weight change.

For both analyses, missing ELF scores at baseline $(n=5)$ and weight at follow-up $(n=4)$ were imputed using multiple imputation by chained equations with predictive mean matching (5 imputations and 100 iterations). The sensitivity analysis included only complete cases. We also conducted an independent-sample $t$ test on the changes of ELF among those who lost less than or at least $10 \%$ of their weight, as a $10 \%$ weight loss has been associated with histological fibrosis regression [20]. An outlier that was $>3$ SDs from the mean was excluded from the $t$ test, but exclusion of the outlier from the regression models did not materially affect the estimates. Analysis was conducted in R, v3.5.0. 
Koutoukidis et al.: Weight Loss on Enhanced Liver Fibrosis Score

Table 1. Baseline characteristics of the cohort

\begin{tabular}{lccc}
\hline & Standard care & $\begin{array}{c}\text { Community weight } \\
\text { loss groups }\end{array}$ & Total \\
\hline$N$ & & 45 & 73 \\
Female sex & 28 & $42(93.3)$ & $70(95.9)$ \\
Ethnicity & $28(100)$ & & \\
$\quad$ White & $27(96.4)$ & $42(93.3)$ & $69(94.5)$ \\
$\quad$ Black/mixed & $1(3.6)$ & $3(6.7)$ & $4(5.5)$ \\
Type 2 diabetes & $1(3.6)$ & $2(4.4)$ & $3(4.1)$ \\
Age, years & $55.19(10.23)$ & $52.9(13.46)$ & $53.78(12.29)$ \\
Weight, kg & $82.02(2.44)$ & $83.21(2.64)$ & $82.75(2.55)$ \\
BMI, kg/m & $31.25(2.44)$ & $31(2.64)$ & $31.1(2.55)$ \\
Glucose, mmol/L* & $4.95(0.83)$ & $4.8(0.7)$ & $4.9(0.8)$ \\
\hline Insulin, pmol/L & $50.03(27.37)$ & $53.62(32.25)$ & $52.24(30.32)$ \\
HbA1c, \% & $5.66(0.3)$ & $5.74(0.44)$ & $5.71(0.39)$ \\
HOMA2-IR & $5.88(2.92)$ & $6.26(3.4)$ & $6.12(3.21)$ \\
Total cholesterol, mmol/L & $5.59(1.06)$ & $5.56(1.22)$ & $5.57(1.15)$ \\
LDL cholesterol, mmol/L* & $3.65(1.25)$ & $3.2(1.5)$ & $3.3(1.4)$ \\
HDL cholesterol, mmol/L & $1.4(0.39)$ & $1.54(0.44)$ & $1.48(0.42)$ \\
Triglycerides, mmol/L* & $1.44(0.85)$ & $1.2(0.6)$ & $1.3(0.8)$ \\
ELF score & $9.03(0.78)$ & $8.88(1.11)$ & $8.93(0.99)$ \\
$\quad$ HA & $52.17(44.36)$ & $51.97(66.8)$ & $52.05(58.86)$ \\
PIIINP & $8.8(3.03)$ & $9.41(4.51)$ & $9.17(4)$ \\
TIMP1 & $210.32(58.41)$ & $198.61(53.55)$ & $203.1(55.36)$ \\
\hline
\end{tabular}

Data are presented as $n(\%)$ or mean (SD), unless otherwise indicated. * Data are presented as median (interquartile range). ELF, enhanced liver fibrosis; HA, hyaluronic acid; PIIINP, amino-terminal propeptide of type III procollagen; TIMP-1, tissue inhibitor of metalloproteinase 1.

\section{Results}

Demographic, anthropometric, and biochemical markers were similar between the intervention and comparator groups (Table 1). The mean (SD) BMI of participants was 31.10 (2.55) and the mean (SD) ELF score at baseline was 8.93 (0.99) indicating moderate fibrosis, with 3 participants (4\%) having an ELF score above the cut-off of 10.51 for advanced fibrosis. At baseline, BMI did not correlate with the ELF score $(r=0.20, p=0.09)$. Percentage weight change at 12 months in the intervention group was $-9.0 \%$ (5.9) and in the usual care group was $-5.8 \%$ (5.4) (between-group difference: $-2.66 \mathrm{~kg}$, 95\% CI: -5.02 to -0.30 ). Compared with baseline, the ELF score decreased by $-0.05(0.99)$ points in the intervention group and $-0.25(0.75)$ points in the usual care group. There was no evidence of a significant effect of the intervention on the ELF score (between-group difference: +0.13 ( $95 \%$ CI: -0.25 to 0.52 , $p=0.50$ ). The interaction between treatment and baseline ELF score was not significant $(-0.02$ (95\% CI: -0.56 to $0.52, p=0.93)$.

Percentage weight change in whole cohort was -7.8\% (5.9) and the change in ELF was $-0.12(0.90)$ points. There was no evidence of an association between weight change and change in ELF; the coefficient for a $5 \%$ reduction in weight was -0.15 (95\% CI: -0.30 to $0.0002, p=0.056$ ). There was a significant interaction between weight change and the ELF score at baseline such that people with higher baseline ELF scores reduced while those with lower scores increased at 12-month follow-up ( $p=0.002$ for interaction) (Fig. 1). There was no significant difference in changes in ELF in those who lost at least $10 \%$ of their weight compared with those who lost less than $10 \%(p=0.14)$. Changes in insulin resistance 
Fig. 1. Median (interquartile range) changes in the Enhanced Liver Fibrosis score in the whole cohort by percentage of achieved weight loss at 1 year. Values above the yellow and red lines indicate cut-off values for possible moderate and severe liver fibrosis, respectively.

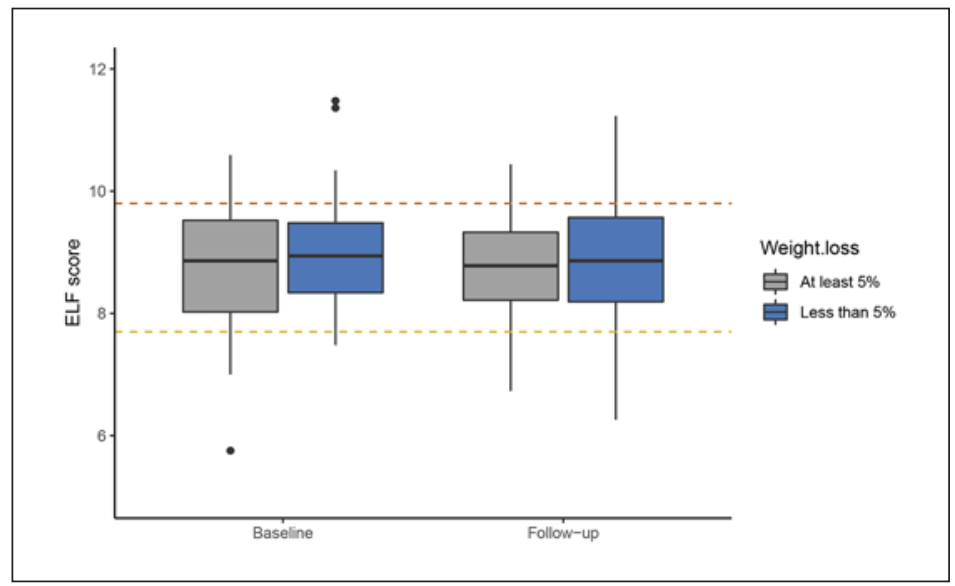

(HOMA2-IR) were not associated with the ELF score at 12 months $(-0.001,95 \% \mathrm{CI}:-0.01$ to 0.002 ).

These findings were essentially unchanged in a complete cases sensitivity analysis. There was no evidence of an effect of the intervention on the ELF score (between-group difference: 0.06 points, $95 \% \mathrm{CI}:-0.33$ to $0.45, n=68$ ). Weight change in the whole cohort was not associated with changes in the ELF score $(-0.1,95 \% \mathrm{CI}:-0.25$ to $0.002, n=64)$.

\section{Discussion}

\section{Summary}

In this secondary analysis of a randomised controlled trial, we found no evidence of an effect of a community weight loss programme on changes in the ELF score and no association between weight loss and the ELF score in people who had, on average, an ELF score compatible with moderate fibrosis.

\section{Strengths and Limitations}

To our knowledge, this is the first study to quantify expected changes in the ELF score following weight loss through lifestyle modification. The confidence intervals were precise enough to probably exclude significant differences in ELF following weight loss through lifestyle modification in this population. Strengths of the study include the randomised controlled design and long-term follow-up. However, the results of this study should be interpreted with caution given the small sample size and lack of assessment of changes in other liver biomarkers, such as alanine transaminase or blood markers of liver fibrosis such as the NAFLD fibrosis score and the fibrosis-4 (FIB-4) score. As progression of liver fibrosis is slow, 1 year is the minimum recommended follow-up to detect fibrosis changes [21]. Fibrosis improved at 1 year in a large single-arm weight loss trial in NASH [20], although there was no evidence of changes in fibrosis in randomised trials with smaller samples [22]. Most participants in our study consumed some alcohol, but none had a diagnosis indicative of alcohol abuse. We did not screen specifically for NAFLD or other liver disease, such as hepatitis. However, the prevalence of hepatitis B and C infection is $<1 \%$, so it is not likely to be quantitatively important in these results. Furthermore, the population studied here was affected by overweight, and the liver biomarker showed some evidence of liver dysfunction, such that NAFLD is the most likely diagnosis. 


\section{Comparison with Existing Literature}

Secondary analysis of a previous trial of a novel agent to treat NASH showed that people who lost $5 \%$ of their BMI had an improvement in another non-invasive biomarker of liver fibrosis: liver stiffness measured with magnetic resonance elastography [23]. However, it is not clear whether this is an effect of weight loss per se or confounded by specific effects of the pharmacotherapy on fibrosis. In a systematic review and meta-analysis, weight loss interventions in people with NAFLD and NASH led to similar weight loss as in the current study and in improvement in biopsy-proven steatosis and the NAFLD activity score [22]. Furthermore, the direction of the association between weight loss interventions and biopsy-proven liver fibrosis went in the expected direction, albeit not significant (change in fibrosis score: -0.13 , $95 \%$ CI: -0.54 to 0.27 ), and we would have expected to see a reduction in fibrosis if the estimate were more precise.

\section{Implications for Research and/or Practice}

Using the ELF test to assess weight loss treatment efficacy in improving liver fibrosis may be of limited value and, until validated non-invasive biomarkers are available, biopsy remains the gold-standard assessment for liver fibrosis. However, given the invasive nature of liver biopsies, research on the validation of the ELF and other low-risk, low-cost tests is critical as this can facilitate follow-up of patients with liver fibrosis and population-based studies on disease progression. Although assessment of the ELF score is currently limited in primary care [17], its use should be expected to rise, given the current NICE guidance and its utility in primary- to secondary-care NAFLD referrals $[13,14]$. Future weight loss trials should examine the effect of lifestyle interventions specifically in people with a biopsy-proven fibrosis. Followup data after bariatric surgery could also be used to test if larger weight loss might reduce ELF score. Genetic and epigenetic factors, inflammation, comorbidities, and the gut microbiota are also implicated in fibrosis progression in NAFLD [24, 25]. These were balanced in our randomised comparison but should be carefully accounted for in future observational studies.

In conclusion, we found no evidence that the ELF score meaningfully changed in an unselected population with overweight with moderate fibrosis following a lifestyle intervention with modest weight loss.

\section{Acknowledgment}

We thank the co-investigators in this trial, especially Amy Ahern, Hans Hauner, and Ian Caterson; as well as Sarah Casey, Veronica Bell, and Raakesh Modi for administrative and technical support. We also thank the participants in the study for their willingness to take part and allow their samples to be used for these further analyses. The data sets used and/or analysed during the current study and the statistical code are available from the corresponding author on reasonable request.

\section{Statement of Ethics}

This study received ethics approval from Nottingham Research Ethics Committee, UK (Ref. 07/Q2404/40), the ethical committee of the Faculty of Medicine of the Technische Universität München, Germany (Ref. 1812/07), and the ethics review committee (Royal Prince Alfred Hospital zone) of the Sydney South West Area Health Service, Australia (Ref. X07-0089). All patients provided written informed consent. 
Koutoukidis et al.: Weight Loss on Enhanced Liver Fibrosis Score

\section{Disclosure Statement}

N.M.A., P.A., and S.A.J. are investigators on a trial funded by Cambridge Weight Plan UK, Ltd, but have received no personal payments from this or any other private company. No other conflicts of interest are reported.

\section{Funding Sources}

The trial reported here was funded by Weight Watchers through an investigator-led grant awarded to S.A.J. This work was funded by the National Institute for Health Research (NIHR) Oxford Biomedical Research Centre (grant number: IS-BRC-1215-20008). S.A.J. and P.A. are NIHR Senior Investigators and also funded by the Oxford NIHR Collaboration and Leadership in Applied Health Research. The funder had no role in the design and conduct of the study; collection, management, analysis, and interpretation of the data; preparation, review, or approval of the manuscript; and decision to submit the manuscript for publication. The views expressed are those of the authors and not necessarily those of the NHS, the NIHR, or the Department of Health and Social Care.

\section{Author Contributions}

All authors contributed to the conception, design, analysis, and interpretation of the data. D.A.K. drafted the manuscript, and all authors critically revised and approved the final version of the manuscript.

\section{References}

1 Younossi Z, Anstee QM, Marietti M, Hardy T, Henry L, Eslam M, et al. Global burden of NAFLD and NASH: trends, predictions, risk factors and prevention. Nat Rev Gastroenterol Hepatol. 2018 Jan;15(1):11-20.

2 Polyzos SA, Kountouras J, Mantzoros CS. Obesity and nonalcoholic fatty liver disease: from pathophysiology to therapeutics. Metabolism. 2019 Mar;92:82-97.

3 Leung JC, Loong TC, Wei JL, Wong GL, Chan AW, Choi PC, et al. Histological severity and clinical outcomes of nonalcoholic fatty liver disease in nonobese patients. Hepatology. 2017 Jan;65(1):54-64.

4 Abenavoli L, Milic N, Di Renzo L, Preveden T, Medić-Stojanoska M, De Lorenzo A. Metabolic aspects of adult patients with nonalcoholic fatty liver disease. World J Gastroenterol. 2016 Aug;22(31):7006-16.

5 Targher G, Byrne CD, Lonardo A, Zoppini G, Barbui C. Non-alcoholic fatty liver disease and risk of incident cardiovascular disease: A meta-analysis. J Hepatol. 2016 Sep;65(3):589-600.

6 Dyson J, Jaques B, Chattopadyhay D, Lochan R, Graham J, Das D, et al. Hepatocellular cancer: the impact of obesity, type 2 diabetes and a multidisciplinary team. J Hepatol. 2014 Jan;60(1):110-7.

7 Williams R, Aspinall R, Bellis M, Camps-Walsh G, Cramp M, Dhawan A, et al. Addressing liver disease in the UK: a blueprint for attaining excellence in health care and reducing premature mortality from lifestyle issues of excess consumption of alcohol, obesity, and viral hepatitis. Lancet. 2014 Nov;384(9958):1953-97.

8 Angulo P, Kleiner DE, Dam-Larsen S, Adams LA, Bjornsson ES, Charatcharoenwitthaya P, et al. Liver Fibrosis, but No Other Histologic Features, Is Associated With Long-term Outcomes of Patients With Nonalcoholic Fatty Liver Disease. Gastroenterology. 2015;149:389-397.e310.

9 Ekstedt M, Hagström H, Nasr P, Fredrikson M, Stål P, Kechagias S, et al. Fibrosis stage is the strongest predictor for disease-specific mortality in NAFLD after up to 33 years of follow-up. Hepatology. 2015 May;61(5):154754.

10 Sumida Y, Nakajima A, Itoh Y. Limitations of liver biopsy and non-invasive diagnostic tests for the diagnosis of nonalcoholic fatty liver disease/nonalcoholic steatohepatitis. World J Gastroenterol. 2014 Jan;20(2):47585.

11 Guha IN, Parkes J, Roderick P, Chattopadhyay D, Cross R, Harris S, et al. Noninvasive markers of fibrosis in nonalcoholic fatty liver disease: validating the European Liver Fibrosis Panel and exploring simple markers. Hepatology. 2008 Feb;47(2):455-60. 
Koutoukidis et al.: Weight Loss on Enhanced Liver Fibrosis Score

12 Staufer K, Halilbasic E, Spindelboeck W, Eilenberg M, Prager G, Stadlbauer V, et al. Evaluation and comparison of six noninvasive tests for prediction of significant or advanced fibrosis in nonalcoholic fatty liver disease. United European Gastroenterol J. 2019 0ct;7(8):1113-23.

13 National Institute for Health and Care Excellence. Non-alcoholic fatty liver disease (NAFLD): assessment and management. London: National Institute for Health and Care Excellence; 2016.

14 Srivastava A, Gailer R, Tanwar S, Trembling P, Parkes J, Rodger A, et al. Prospective evaluation of a primary care referral pathway for patients with non-alcoholic fatty liver disease. J Hepatol. 2019 Aug;71(2):371-8.

15 Goldschmidt I, Di Nanni A, Streckenbach C, Schnell K, Danne T, Baumann U. Improvement of BMI after Lifestyle Intervention Is Associated with Normalisation of Elevated ELF Score and Liver Stiffness in Obese Children. BioMed Res Int. 2015;2015:457473.

16 Armstrong MJ, Gaunt P, Aithal GP, Barton D, Hull D, Parker R, et al.; LEAN trial team. Liraglutide safety and efficacy in patients with non-alcoholic steatohepatitis (LEAN): a multicentre, double-blind, randomised, placebo-controlled phase 2 study. Lancet. 2016 Feb;387(10019):679-90.

17 Sheridan DA, Aithal G, Alazawi W, Allison M, Anstee Q, Cobbold J, et al. Care standards for non-alcoholic fatty liver disease in the United Kingdom 2016: a cross-sectional survey. Frontline Gastroenterol. 2017 Oct;8(4): 252-9.

18 Jebb SA, Ahern AL, Olson AD, Aston LM, Holzapfel C, Stoll J, et al. Primary care referral to a commercial provider for weight loss treatment versus standard care: a randomised controlled trial. Lancet. 2011 Oct;378(9801): 1485-92.

19 Lichtinghagen R, Pietsch D, Bantel H, Manns MP, Brand K, Bahr MJ. The Enhanced Liver Fibrosis (ELF) score: normal values, influence factors and proposed cut-off values. J Hepatol. 2013 Aug;59(2):236-42.

20 Vilar-Gomez E, Martinez-Perez Y, Calzadilla-Bertot L, Torres-Gonzalez A, Gra-Oramas B, Gonzalez-Fabian L, et al. Weight Loss Through Lifestyle Modification Significantly Reduces Features of Nonalcoholic Steatohepatitis. Gastroenterology. 2015;149:367-378.e365; quiz e314-365.

21 Sanyal AJ, Brunt EM, Kleiner DE, Kowdley KV, Chalasani N, Lavine JE, et al. Endpoints and clinical trial design for nonalcoholic steatohepatitis. Hepatology. 2011 Jul;54(1):344-53.

22 Koutoukidis DA, Astbury NM, Tudor KE, Morris E, Henry JA, Noreik M, et al. Association of Weight Loss Interventions With Changes in Biomarkers of Nonalcoholic Fatty Liver Disease: A Systematic Review and Metaanalysis. JAMA Intern Med. 2019 Jul;179(9):1262.

23 Patel NS, Hooker J, Gonzalez M, Bhatt A, Nguyen P, Ramirez K, et al. Weight Loss Decreases Magnetic Resonance Elastography Estimated Liver Stiffness in Nonalcoholic Fatty Liver Disease. Clin Gastroenterol Hepatol. 2017 Mar;15(3):463-4.

24 Pingitore P, Dongiovanni P, Motta BM, Meroni M, Lepore SM, Mancina RM, et al. PNPLA3 overexpression results in reduction of proteins predisposing to fibrosis. Hum Mol Genet. 2016 Dec;25(23):5212-22.

25 Schuppan D, Surabattula R, Wang XY. Determinants of fibrosis progression and regression in NASH. J Hepatol. 2018 Feb;68(2):238-50. 